\title{
Metastatic hepatocellular carcinoma to the parotid gland Associated with multiple metastasis: Case report
}

\section{Mingxia Sun}

Henan Provincial People's Hospital

Shundong Cang ( $D$ 13027120395@163.com)

Henan Provincial People's Hospital https://orcid.org/0000-0002-4115-8686

\section{Research}

Keywords: hepatocellular carcinoma, parotid gland metastasis

Posted Date: August 18th, 2020

DOI: https://doi.org/10.21203/rs.3.rs-55423/v1

License: (c) (1) This work is licensed under a Creative Commons Attribution 4.0 International License.

Read Full License 


\section{Abstract}

Introduction: The incidence of liver cancer is very high in China, and it is more common in patients with a history of hepatitis B.Most patients with liver cancer have distant metastasis at the time of visiting, but parotid metastasis is very rare. We report a patient with parotid enlargement who was diagnosed with multiple metastasis of liver cancer.

Presentation of Case: A 52-year-old male presented with parotid enlargement for one month after the right deep maxillofacial neck mass was cut and biopsied,Metastatic hepatocellular carcinoma of the parotid gland associated with multiple metastasis was diagnosed.

Discussion: Metastasis of liver cancer to the parotid gland is very rare, to date, only 7 cases HCC metastasis to the parotid gland have been reported, this case is the first report in China. For patients with parotid enlargement at the first diagnosis, no history of hepatitis or liver cancer, and normal or slightly elevated AFP,It is very easy to misdiagnose and miss diagnosis.Pathological diagnosis and complete systemic examination are very important in the diagnosis of tumor.Although very rare, parotid metastases in HCC should be maintained with high suspicion.

\section{Background}

Hepatocellular carcinoma (HCC) is one of the most common cancers worldwide, with about 841,000 diagnoses and 782,000 deaths per year[1]. it is estimated to be responsible for 746,000 deaths per year (9\% of the total deaths from cancer)[2].The most common risk factor for HCC is chronic HBV infection, which accounts for more than $50 \%$ of all cases globally and $60-80 \%$ in some Asian countries such as China, Korea, and Vietnam [3].HCV, HBV, can directly lead to HCC without the presence of cirrhosis.Some patients with liver cancer have alcoholic hepatitis or a history of heavy drinking[4-7].Alpha-fetoprotein (AFP) is the most frequent used serological marker,AFP is an important index for the diagnosis of primary liver cancer. Elevated AFP level can be used as the basis for the clinical diagnosis of liver cancer,but some patients with liver cancer have normal AFP[8].in addition to AFP, histopathological biopsy is the gold standard for the diagnosis of liver cancer[9].The common sites of liver cancer metastasis include intrahepatic metastasis, lung, peritoneum, bone, adrenal gland, etc. The prognosis for liver cancer is very poor,Patients with metastases have worse prognosis.Liver cancer rarely metastases to other than common sites like the salivary glands. Metastasis to rare sites of liver cancer is easily to be ignored.This paper reports a case of parotid gland metastasis of hepatocellular carcinoma. This patient was first treated for facial mass and parotid gland enlargement, The patient complained that he had no history of hepatitis, the examination after admission indicated hepatitis $B$, with a history of small amount of alcohol consumption, and a slight increase in AFP. It was difficult to consider the diagnosis of liver cancer in this case, but the parotid lesion was excised and pathologically confirmed as metastatic liver cancer.Parotid gland tumors can be classified as benign and malignant, and malignant tumors can be divided as primary or metastatic. The primary foci of metastatic parotid gland carcinoma are mostly from the head and neck, the liver of metastatic carcinoma in the parotid gland origin is very rare, and easy to 
be ignored, thus increasing the difficulty of diagnosis and treatment and easily delaying the treatment of patients.especially when the AFP is normal or no history of hepatitis, patients are less likely to consider the transfer of liver cancer.histopathologic diagnosis is particularly important in the diagnosis of the tumor, including excision biopsy, biopsy.So far, a total of only 7 English literatures have reported parotid gland metastasis of liver cancer, which further suggested the particularity of this situation [10-16]. We here report an additional case of this rare association.

\section{Case Report}

A 52-year-old male presented with a progressively enlarging no painful right facial swelling for 1 months. Physical examination revealed right-sided swelling in the face of about $5 \times 5 \mathrm{~cm}$ on the parotid gland that was firm.and later pain appeared,After the use of amoxicillin and other anti-inflammatory drugs, the pain was relieved, but the tumor gradually increased, accompanied by increased pain, with numbness and discomfort of the right lip. He also reported a history of significant weight loss and progressive abdominal distention and discomfort.No family history of cancer. The previous history of hepatitis $b$ was unknown, and the patient was diagnosed as hepatitis $b$ during the first diagnosis and hospitalization,No signs of cirrhosis.After diagnosis, patients were regularly treated with entecavir orally. With a drinking

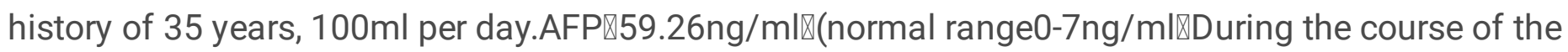
disease, the highest value of AFP is $132.8 \mathrm{ng} / \mathrm{ml}, \mathrm{CA} 125 \square \mathrm{CA} 199 \square \mathrm{CA} 724 \llbracket \mathrm{CEA}$ are all normal.computed

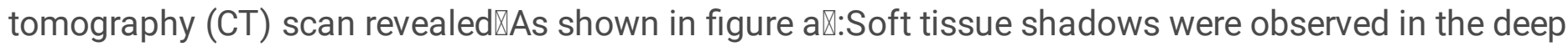
part of the right parotid gland, with a size of about $59 \mathrm{~mm} \star 57 \mathrm{~mm} \star 45 \mathrm{~mm}$. The enhancement scan showed obvious uneven enhancement. Destruction shadows were observed in the adjacent bone, the peripharyngeal wall was thickened and the pharyngeal space was narrowed, and no obvious enlarged lymph nodes were observed in the neck. Multiple nodules in both lungs were considered as metastatic tumors.Multiple mediastinal lymph nodes were enlarged, metastasis was considered, and the right lobe of the liver was occupied.Right adrenal nodule, metastatic possibility.The right deep maxillofacial neck mass was cut and biopsied. During the surgery, it was found that $₫ A$ s shown in figure $b \rrbracket$ : the tumor was located in the deep lobe of the right parotid gland and the deep muscle of the right masseter, with complete envelopment, white content, loose tissue, crispy fish-like tissue, and abundant blood supply.Pathological diagnosis: right facial malignant tumor, which was consistent with hepatocellular carcinoma metastasis in combination with HE morphology and immunohistochemistry. The size was $1.8 * 1.2 * 0.5 \mathrm{~cm}$, and the immunohistochemistry structure suggested: ck8/18 (+), arginase- $1(+)$, glypican-3 $(-)$, Hepatocyte $(+)$, Villin(focal +), Calponin(-), CD117(-), CEA (-), CK (AE1 / AE3) (-), CK7 (focal point +$)$, the DOG (+) - 1, EMA (+), Ki67 (about + 70\%), about (-), S - 100 (-), SMA (-). The case was diagnosed by imaging and pathology as metastatic hepatocellular carcinoma to the parotid gland associated with multiple metastasis. The tumor recurred in the postoperative site of parotid gland 22 days after surgery. CT indicated that soft tissue shadow was seen in the deep part of right parotid gland, with the size of about $59 \mathrm{~mm} \star 57 \mathrm{~mm} \star 45 \mathrm{~mm}$. The patient was then given targeted therapy orally with sorafenib.The parotid gland lesion was significantly reduced after one month, efficacy evaluation is Partial response(PR). The lesion developed again after continued oral administration of sorafenib for 2 months, 
efficacy evaluation is progressive disease(PD).and replacement of apatinib targeted therapy were given. The disease progressed again after 5 months of oral administration of apatinib, The patient was treated with traditional Chinese medicine and died six months later.

\section{Conclusions}

This paper reports a case report of a male patient with IV-stage hepatocellular carcinoma (stage D according to Barcelona Clinic of Liver Cancer, BCLC, staging classification) with right parotid gland metastasis, complicated with lung metastasis, Multiple mediastinal lymph nodes and the right lobe of the liver,Right adrenal nodule metastasis.which is the first case reported in China.The patient was treated for parotid gland enlargement, and the pathological diagnosis was made by parotid gland tissue resection and pathological biopsy.Ki67 (about $+70 \%$ ),AFP is slight increase, the patient was diagnosed hepatitis B for the first time during the first diagnosis and hospitalization. The tumor recurred in the postoperative site of parotid gland 22 days after surgery. The parotid gland lesion decreased after 1 month of oral administration of sorafenib, and increased again after 2 months of the targeted drugs.After the disease progressed, apatinib was switched to targeted therapy, and the disease progressed again after 5 months under control.Overall survival is nearly 15 months. We report an additional case of the rare metastasis of hepatocellular carcinoma to the parotid glands. Furthermore, the present case demonstrates the importance of pathological diagnosis and general systemic examination for obtaining an accurate final diagnosis.

\section{Discussion}

Hepatocellular carcinoma (HCC) is the fourth leading cause of cancer-related death worldwide[17].Despite advances in its treatment, liver cancer remains one of the most difficult cancers to treat. For patients with early HCC, local ablation, surgical resection or liver transplantation provide curative potential. However, recurrence of HCC remains a major problem after curative treatment.Treatment of advanced hepatocellular carcinoma (HCC) remains a challenge. Although several antiangiogenic targeted agents and checkpoint inhibitors have been recently approved for the treatment of HCC, the objective tumor response rates of these agents are in the range of $10 \%$ to $24 \%[17,18]$.In view of the limited therapeutic effect of liver cancer, early detection and diagnosis and treatment of liver cancer patients are of great importance.Since parotid gland metastasis of hepatocellular carcinoma is very rare, it is already at an advanced stage at the time of diagnosis. If there is a missed or misdiagnosed situation that cannot be detected in time, and the patient cannot receive timely and effective treatment, the overall survival and prognosis of the patient will be worse. This report and the past Relevant case reports suggest the existence of this phenomenon, which has resulted in earlier diagnosis and treatment for patients, which has brought benefits for patient.

Several meta-analyses have demonstrated that the risk of HCC is 15-20 times greater among HBVinfected individuals compared with the uninfected population[19]. with increasing awareness of reporting and better screening services, China have reported increasing incidence of HCC for males and females 
[20]. Despite the high incidence of liver cancer throughout China, a decreasing trend has been observed in some regions because of neonatal vaccination for HBV infection[20].Effective and potentially long-term antiviral therapy should be started in all patients with chronic hepatitis B infection and active liver disease.The use of hepatitis $B$ vaccine and antiviral treatment can better prevent and control the occurrence and development of liver cancer.The case of this article found hepatitis $B$ when the liver cancer was diagnosed, at this time there were distant metastases. The stage was advanced and the prognosis was poor.Unfortunately, the patient had not been examined for hepatitis B before, and had no prior antiviral treatment,which also reminds us of the importance of physical examination and early prevention. For hepatitis patients, we need to be more alert to the occurrence of liver cancer.

Hepatocellular carcinoma (HCC) commonly metastasizes to the lungs, lymph nodes, peritoneum, adrenal glands, and bone, although there are uncommon reports of metastases to the ovaries, skin, heart, pharynx, orbit, and external auditory canal [21--26]. Metastasis to parotid glands is rare and to the best of our knowledge only 7 cases of hepatocellular carcinoma metastatic to parotid glands have been reported since 1998 [10-16], suggesting that this association is rare.If the patient has a history of liver cancer, parotid gland enlargement is more likely to indicate the possibility of liver cancer metastasis.but If the patient is first diagnosed because of parotid gland enlargement,espicially when the patient with no history of hepatitis, alcoholic liver or liver cancer, and with normal or slightly elevated AFP, which made the diagnosis of liver cancer more difficult and easy to be misdiagnosed, It is easy to cause misdiagnosis and missed diagnosis when the diagnosis of HCC was not clinically suspected,because there are no highrisk factors for liver cancer.Especially, The primary site of parotid gland metastasis is mostly located in the head and neck region,metastatic localizations from liver cancer are even more rare. which should arouse the vigilance of the medical staff.

Although CT, Magnetic Resonance Imaging(MRI), ultrasound and other examinations play an important role in diagnosing the nature of parotid gland masses and tumor staging, and have guiding significance for treatment after diagnosis, the diagnosis of histopathology is still very important,oncologists often use pathological diagnosis to determine the nature and type of the tumor.Biopsy for parotid gland tumors include fine needle aspiration biopsy and parotidectomy. There are reports that fine needle aspiration biopsy of parotid gland in the diagnosis of malignancy with the sensitivity of $54-92 \%$, adn specificity in the range of $86-100 \%$.there are false-negative and the false-positive[27,28].Excision biopsy of parotid gland is the other method of diagnosis. It cannot be used for treatment of patients with liver metastases. However, because tissue samples are more abundant than fine needle biopsy, it is more helpful for accurate pathological diagnosis.In this case, the pathology was confirmed by parotidectomy biopsy,After the parotid gland tumor was removed, the patient's parotid gland recurred quickly.

As the prognosis of HCC depends largely on the stage at which the tumor is detected, detection of HCC early in its development is critical to improve the survival of affected patients [29]. The treatment of patients with advanced liver cancer is very important.Sorafenib and lenvatinib were the target therapy that improved overall survival (OS) for unresectable HCC (uHCC) [30-32]. For patients who progress after failure of first-line treatment,subsequent systemic anticancer medication could be an important treatment 
that may lead to longer OS.Apatinib is a novel receptor tyrosine kinase inhibitor selectively targeting VEGFR-2. It is an orally-bioavailable agent currently being studied in several solid tumour types[33].In addition to the targeted therapy,A number of ongoing clinical trials related checkpoint inhibitors aiming to improve the therapeutic effect of liver cancer patients[34].The first-line treatment of this patient is sorafenib,progressed after 2 months of the targeted drugs.After switching to apatinib, the condition was controlled for 5 months, and then the condition progressed again.Later, the patient chose traditional Chinese medicine for treatment,died six months later.

The treatment effect of advanced liver cancer is limited, and the overall survival time is short. Most patients have a history of hepatitis, without systemic antiviral treatment, and some patients with normal AFP level or Slightly elevated AFP level, which is easy to be ignored. When liver cancer is diagnosed, it is already in the advanced stage, resulting in low overall treatment efficiency. This case report is very meaningful. 1. Hepatitis B was found in the patient's first visit. He has an unknown history of hepatitis. However, it is not ruled out that the patient has hepatitis in the past but is unknown and has not been diagnosed and treated. 2. The patient's first visit to the Department of Oral Surgery, AFP level is not significantly increased,the first physician did not consider the possibility of Parotid gland metastasis of liver cancer, and performed local resection based on the primary tumor of the parotid gland, and did not give timely treatment for liver cancer after the operation. After the tumor recurred at the parotidectomy site, the patient was transferred to the oncology department for liver cancer related treatment. More than 20 days after the postoperative pathological diagnosis of this patient, systemic anti-tumor therapy for liver cancer was started. 3. Oncologists perform treatment according to the diagnosis of histopathology, suggesting the importance of pathological diagnosis in tumor diagnosis. This case suggests that our clinicians have insufficient knowledge about the metastasis of rare parts of liver cancer, such as parotid gland metastasis,which easily delays the diagnosis and treatment of patients. This case is the eighth reported case of metastatic hepatocellular carcinoma to the parotid gland. It is hoped that more and more reports of rare cases can attract the attention of clinicians. For early prevention, early detection and treatment of patients provide more help.

\section{Abbreviations}

Hepatocellular carcinoma (HCC),Alpha-fetoprotein (AFP),Computed tomography (CT),Partial response(PR),Magnetic Resonance Imaging(MRI)

\section{Declarations}

\section{Ethics approval and consent to participate:}

This article has the consent of the patient's family.

\section{Consent for publication:}


The author(s) read and approved the final manuscript.

\section{Availability of data and materials:}

All information of this patient during this case report are included in the published article and its supplementary information files.

\section{Competing interests:}

The authors declare that they have no competing interests.

\section{Funding:}

This work was supportted by The Project of Henan Province Health and Family Planning Innovative Talents("51282" project leading talent)

\section{Authors' contributions:}

Sun Mingxia formulated the research question, initiated thestudy,conducted case collection and summary,carried out literature search;Cang Shundong wrote,reviewed and edited the manuscript.Acknowledgements:We are grateful to Editorial Office of Orphanet Journal of Rare Diseases for responding to our e-mail and providing additional follow-up.

\section{References}

1.Bray F, Ferlay J, Soerjomataram I, Siegel RL, Torre LA, Jemal A. Global cancer statistics 2018: GLOBOCAN estimates of incidence and mortality worldwide for 36 cancers in 185 countries. CA Cancer J Clin.2018 Nov;68(6): 394-424.

2.Ferlay J, Soerjomataram I, Ervik M, Dikshit R, Eser S, Mathers C, et al. GLOBOCAN 2012 v1.0, Cancer Incidence and Mortality Worldwide: IARC CancerBase No. 11 [Internet]. Lyon, France: International Agency for Research on Cancer; 2013. Available at: http://globocan.iarc.fr. Accessed: December 15, 2014.

3.de Martel C, Maucort-Boulch D, Plummer M, Franceschi S. World-wide relative contribution of hepatitis B and $C$ viruses in hepatocellular carcinoma. Hepatology 2015;62:1190-1200

4.Massarweh N N , El-Serag H B . Epidemiology of Hepatocellular Carcinoma and Intrahepatic Cholangiocarcinoma[J]. Cancer Control, 2017, 24(3):107327481772924. 
5.Ott JJ, Stevens GA, Groeger J, Wiersma ST. Global epidemiology of hepatitis B virus infection: new estimates of age-specific HBsAg seroprevalence and endemicity. Vaccine. 2012;30(12):2212-2219.

6.Shiani A , Narayanan S, Pena L , et al. The Role of Diagnosis and Treatment of Underlying Liver Disease for the Prognosis of Primary Liver Cancer[J]. Cancer Control, 2017, 24(3):107327481772924.

7.Llovet JM, Zucman-Rossi J, Pikarsky E, et al. Hepatocellular carcinoma. Nat Rev Dis Primers. 2016;2:16018. Published 2016 Apr 14.

8.Dimitroulis D, Damaskos C, Valsami S, et al. From diagnosis to treatment of hepatocellular carcinoma: An epidemic problem for both developed and developing world. World J Gastroenterol. 2017;23(29):52825294.

9.Park JH, Kim JH. Pathologic differential diagnosis of metastatic carcinoma in the liver. Clin Mol Hepatol. 2019;25(1):12-20.

10.Elzouki AN, Elkhider H, Yacout K, Al Muzrakchi A, Al-Thani S, Ismail O. Metastatic hepatocellular carcinoma to parotid glands. Am J Case Rep. 2014;15:343-347.

11.Dargent JL, Deplace J, Schneider E et al: Hepatocellular carcinoma metastatic to the parotid gland: initial diagnosis by fine needle aspiration biopsy. Acta Cytol, 1998; 42(3): 824-26 12.Romanas MM, Cherian R, McGregor DH et al: Hepatocellular carcinoma diagnosed by fine-needle aspiration of the parotid gland. Diagn Cytopathol 2004;30(6): 401-5

13.Vitale AR, Compilato D, Coletti $G$ et al: Metastatic hepatocellular carcinoma of the parotid region without lung metastasis: a case report. Int J Oral Maxillofa Surg, 2009; 38(6): 696-98 14.Moore FR, Bergman S, Geisinger KR: Metastatic hepatocellular carcinoma mimicking acinic cell carcinoma of the parotid gland: a case report. ActaCytol, 2010; 54(5 Suppl.): 889-92

15.Yu YD, Kima DS, Jung SW et al: Metastatic hepatocellular carcinoma to the parotid gland: Case report and review of the literature. Intern J Surg Case Report, 2013; 4(1): 76-80

16.Aiyer HM, Deb S, Rawat G. Metastatic hepatocellular carcinoma to the parotid gland: A diagnostic dilemma with review of the literature. Indian J Pathol Microbiol. 2019;62(4):582-585.

17.Shao YY, Li YS, Hsu HW, et al. Potent Activity of Composite Cyclin Dependent Kinase Inhibition against Hepatocellular Carcinoma. Cancers (Basel). 2019;11(10):1433. Published 2019 Sep 26. doi:10.3390/cancers 11101433

18.Yang JD, Hainaut P, Gores GJ, Amadou A, Plymoth A, Roberts LR. A global view of hepatocellular carcinoma: trends, risk, prevention and management. Nat Rev Gastroenterol Hepatol. 2019;16(10):589604. 
19.Shi J, Zhu L, Liu S, Xie WF. A meta-analysis of case-control studies on the combined effect of hepatitis $B$ and $C$ virus infections in causing hepatocellular carcinoma in China. Br J Cancer 2005;92:607-612

20.Omata M, Cheng AL, Kokudo N, et al. Asia-Pacific clinical practice guidelines on the management of hepatocellular carcinoma: a 2017 update. Hepatol Int. 2017;11(4):317-370. doi:10.1007/s12072-0179799-9

21.Kim TH, Cheung DY, Chung WB et al: A case of metastatic hepatocellular carcinoma of the ovary. Korean J Gastroenterol, 2004; 43(3): 215-18

22.Masci G, Magagnoli M, Grimaldi A et al: Metastasis of hepatocellular carcinoma to the heart: a case report and review of the literature. Tumori, 2004; 90(3): 345-47

23.Yamanishi K, Kishimoto S, Hosokawa Y et al: Cutaneous metastasis from hepatocellular carcinoma resembling granuloma teleangiectaticum. J Dermatol, 1989; 16(6): 500-4

24.Oida Y, Ishii M, Dowaki S et al: Hepatocellular carcinoma with metastasis to the pharynx: report of a case. Tokai J Experimental Clin Medicine, 2005; 30(1): 31-34

25.Font RL, Maturi RK, Small RG, Garcia-Rojas M: Hepatocellular carcinoma metastatic to the orbit. Arch Ophthalmol, 1998; 116(7): 942-45

26.Yasumatsu R, Okura K, Sakiyama Y et al: Metastatic hepatocellular carcinoma of the external auditory canal. World J Gastroenterol, 2007; 13(47): 6436-38

27.Fakhry $\mathrm{N}$, Antonini F, Michel $\mathrm{J}$ et al. Fine-needle aspiration cytology in the management of parotid masses: evaluation of 249 patients. Eu Ann Otolaryngol Head Neck Dis 2012;129:131-5. 28. Fakhry N, Santini L, Lagier A, Dessi P, Giovanni A. Fine needle aspiration cytology and frozen section in the diagnosis of malignant parotid tumours. Int J Oral Maxillofac Surg 2014;43:802-5.

29.Singal AG, Pillai A, Tiro J. Early detection, curative treatment, and survival rates for hepatocellular carcinoma surveillance in patients with cirrhosis: a meta-analysis. PLoS Med 2014;11:e1001624.

30.El-Serag HB. Hepatocellular carcinoma. N Engl J Med. 2011 Sep;365(12):1118-27.

31.Llovet JM, Ricci S, Mazzaferro V, Hilgard P, Gane E, Blanc JF, et al.; SHARP Investigators Study Group. Sorafenib in advanced hepatocellular carcinoma. N Engl J Med. 2008 Jul;359(4):378-90.

32.Kudo M, Finn RS, Qin S, Han KH, Ikeda K, Piscaglia F, et al. Lenvatinib versus sorafenib in first-line treatment of patients with unresectable hepatocellular carcinoma: a randomised phase 3 noninferioritytrial. Lancet. 2018 Mar;391(10126):1163-73.

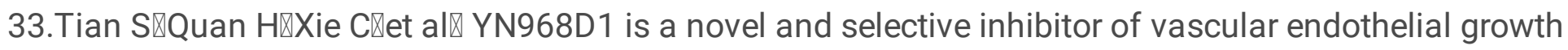

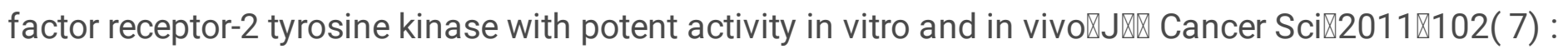


34.Harding JJ, El Dika I, Abou-Alfa GK. Immunotherapy in hepatocellular carcinoma: Primed to make a difference?. Cancer. 2016;122(3):367-377.

\section{Figures}

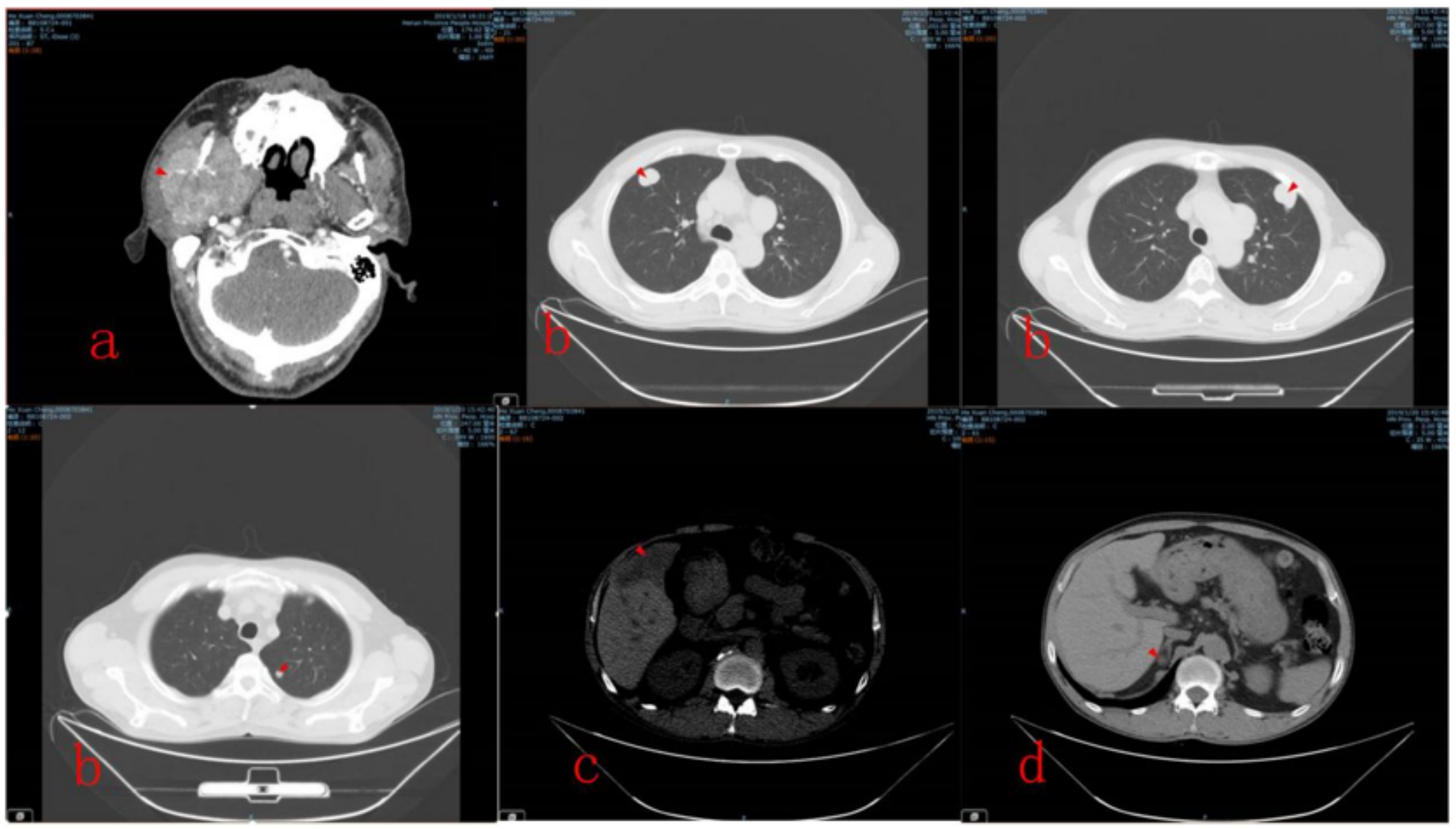

\section{Figure 1}

Figure a. CT indicated (A) deep parotid gland mass, uneven enhancement in contrast-enhanced scan, and proximal bone destruction in the neck; (B) Multiple soft tissue nodules are seen in both lungs; (C) mass shadows were seen in the right lobe of liver, uneven mild enhancement in contrast-enhanced scan; (D) nodular soft mass shadows in the right adrenal gland, and slight enhancement. 


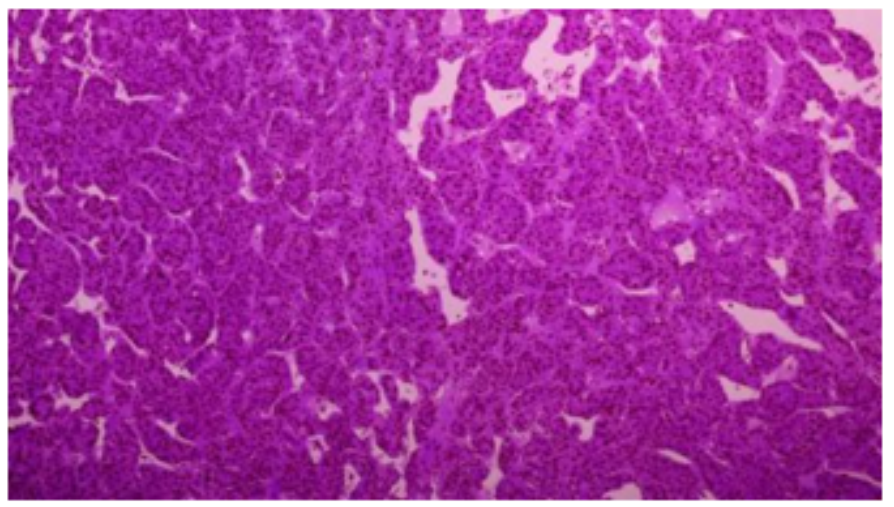

Figure 2

Figure b.Immunohistochemistry suggested (right face) malignant tumor, combined with HE morphology and immunohistochemistry, consistent with hepatocellular carcinoma metastasis. 